\title{
DISCOVERY OF A BRIGHT FIELD METHANE (T-TYPE) BROWN DWARF BY 2MASS
}

\author{
Adam J. Burgasser, ${ }^{1}$ John C. Wilson,${ }^{2}$ J. Davy Kirkpatrick, ${ }^{3}$ Michael F. Skrutskie, ${ }^{4}$ Michael R. Colonno, ${ }^{2}$ \\ Alan T. Enos, ${ }^{2}$ J. D. Smith, ${ }^{2}$ Charles P. Henderson, ${ }^{2}$ John E. Gizis, ${ }^{3}$ \\ MichaEl E. BROWN, ${ }^{5,6}$ AND JAMES R. HOUCK ${ }^{2}$ \\ Received 2000 March 27; accepted 2000 April 17
}

\begin{abstract}
We report the discovery of a bright $(J=13.83 \pm 0.03)$ methane brown dwarf, or $\mathrm{T}$ dwarf, by the Two Micron All Sky Survey. This object, 2MASSI J0559191-140448, is the first brown dwarf identified by the newly commissioned CorMASS instrument mounted on the Palomar 60 inch $(1.5 \mathrm{~m})$ telescope. Nearinfrared spectra from 0.9 to $2.35 \mu \mathrm{m}$ show characteristic $\mathrm{CH}_{4}$ bands at 1.1, 1.3, 1.6, and $2.2 \mu \mathrm{m}$, which are significantly shallower than those seen in other $\mathrm{T}$ dwarfs discovered to date. Coupled with the detection of an $\mathrm{FeH}$ band at $0.9896 \mu \mathrm{m}$ and two sets of $\mathrm{K}$ I doublets at $J$ band, we propose that 2MASS $\mathrm{J} 0559-14$ is a warm $\mathrm{T}$ dwarf, close to the transition between $\mathrm{L}$ and $\mathrm{T}$ spectral classes. The brightness of this object makes it a good candidate for detailed investigation over a broad wavelength regime and at higher resolution.
\end{abstract}

Key words: infrared radiation - stars: fundamental parameters stars: individual (2MASSI J0559191 - 140448) — stars: low-mass, brown dwarfs

\section{INTRODUCTION}

T dwarfs are brown dwarfs that exhibit methane absorption bands at 1.6 and $2.2 \mu \mathrm{m}$ (Kirkpatrick et al. 1999) and thus have effective temperatures $T_{\text {eff }} \lesssim 1200-1300 \mathrm{~K}$ (Fegley \& Lodders 1996; Burrows \& Sharp 1999; Kirkpatrick et al. 2000). The prototype for this class, G1 229B (Nakajima et al. 1995; Oppenheimer 1999), was identified as a cool companion to the nearby M1 V star G1 229A. Recently, seven field objects (Strauss et al. 1999; Burgasser et al. 1999; Cuby et al. 1999; Tsvetanov et al. 2000) and another companion object (Burgasser et al. 2000a) have also been identified as $\mathrm{T}$ dwarfs. The rapid discovery of these cool brown dwarfs has been driven by new sky surveys, such as the Two Micron All Sky Survey (2MASS; Skrutskie et al. 1997) and the Sloan Digital Sky Survey (Gunn \& Weinberg 1995); and deep near-infrared surveys, such as the ESO New Technology Telescope Deep Field (Arnouts et al. 1999).

The $\mathrm{T}$ dwarfs identified to date are remarkably similar to G1 229B, with colors in the range $-0.2 \lesssim J-K_{s} \lesssim 0.2$. Near-infrared spectra are correspondingly similar (Strauss et al. 1999; Burgasser et al. 2000a), likely due to the saturation of $\mathrm{H}_{2} \mathrm{O}$ and $\mathrm{CH}_{4}$ bands that dominate this wavelength regime. Subtle differences in the magnitudes and shapes of

\footnotetext{
${ }^{1}$ Division of Physics, M/S 103-33, California Institute of Technology, Pasadena, CA 91125; diver@its.caltech.edu.

${ }^{2}$ Space Sciences, Cornell University, Ithaca, NY 14853; jcw14@cornell.edu, mrc10@cornell.edu, ate1@cornell.edu, jdsmith@astrosun.tn.cornell.edu, cph5@cornell.edu,jrh13@cornell.edu.

${ }^{3}$ Infrared Processing and Analysis Center, M/S 100-22, California Institute of Technology, Pasadena, CA 91125; davy@ipac.caltech.edu, gizis@ipac.caltech.edu.

${ }^{4}$ Five College Astronomy Department, Department of Physics and Astronomy, University of Massachusetts, Amherst, MA 01003; skrutski@north.astro.umass.edu.

${ }_{5}^{5}$ Division of Geological and Planetary Sciences, M/S 105-21, California Institute of Technology, Pasadena, California 91125; mbrown@gps.caltech.edu.

${ }^{6}$ Alfred P. Sloan Research Fellow.
}

$H$ - and $K$-band flux peaks are observed, because of increased $\mathrm{CH}_{4}, \mathrm{H}_{2} \mathrm{O}$, and $\mathrm{H}_{2}$ collision-induced absorption (CIA) toward cooler effective temperatures (Burgasser et al. 1999; Tsvetanov et al. 2000); and variations in the depths of near-infrared $\mathrm{H}_{2} \mathrm{O}$ and $\mathrm{CH}_{4}$ bands are discerned when compared with Gl 229B (Nakajima et al. 2000; Burgasser et al. 2000a). Nonetheless, the similarity of the near-infrared spectra suggests that either the objects thus far identified are very similar in temperature, around $1000 \mathrm{~K}$ (Marley et al. 1996), or that near-infrared features are fairly insensitive to temperature, making the definition of a $\mathrm{T}$ dwarf spectral sequence in this wavelength regime a difficult proposition, at least at low resolution.

We report the discovery of a $\mathrm{T}$ dwarf by 2 MASS that is unique among its counterparts, as it shows significant differences in its near-infrared features while retaining defining $\mathrm{CH}_{4}$ bands. This object, 2MASSI J0559191-140448 (hereafter 2MASS J0559-14), is also 0.4 mag brighter than G1 229B and more than 1 mag brighter than the field $T$ dwarfs discovered thus far. It is the first brown dwarf to be identified by the newly commissioned Cornell Massachusetts Slit Spectrograph (CorMASS; Wilson et al. 2000), mounted on the Palomar 60 inch $(1.5 \mathrm{~m})$ telescope. In $\S 2$, we discuss the selection of 2MASS J0559-14 from 2MASS data and its spectral identification by CorMASS. In $\S 3$, we discuss the observed spectral features and argue that 2MASS J0559-14 is a warm T dwarf, possibly close to the transition temperature between $\mathrm{L}$ and $\mathrm{T}$ spectral classes. We discuss the brightness of this object and its role in future spectroscopic investigation of the T dwarf class in $\S 4$.

\section{IDENTIFICATION OF 2MASS J0559-14}

\subsection{Selection and Confirmation}

2MASS J0559-14 was initially selected as a $\mathrm{T}$ dwarf candidate from the 2MASS point-source working database. Details on the selection criteria for 2MASS T dwarfs are discussed in Burgasser et al. (2000a). Using the Palomar 60 inch InfraRed camera (IRcam; Murphy et al. 1995), we successfully reimaged this candidate on 1999 September 23 
TABLE 1

Photometric Properties

\begin{tabular}{cccccccc}
\hline \hline Object & \multicolumn{1}{c}{} & & & & & Estimated Distance \\
$(\mathrm{pc})$ & $J-H$ & $H-K_{s}$ & $J-K_{s}$ & \\
\hline 2MASSI J0559191-140448 $\ldots \ldots$ & $13.83 \pm 0.03$ & $13.68 \pm 0.04$ & $13.61 \pm 0.05$ & $0.15 \pm 0.05$ & $0.07 \pm 0.06$ & $0.22 \pm 0.06$ & $5-8^{\mathrm{b}}$ \\
\hline
\end{tabular}

a Source designations for 2MASS sources in the Incremental Release Catalogs are given as "2MASSI Jhhmmss[.]s \pm ddmmss." The suffix conforms to IAU nomenclature convention and is the sexagesimal R.A. and declination at J2000.0 equinox.

${ }^{\mathrm{b}}$ See $\S 4$ for discussion.

(UT) at $J$ band, confirming it as a bona fide candidate (i.e., not a minor planet or artifact). 2MASS J0559-14 is the brightest 2MASS T dwarf candidate confirmed to date, with $J=13.83 \pm 0.03$ (Table 1); it is also the reddest candidate confirmed, with $J-K_{s}=0.22 \pm 0.06$. Optical SERC-EJ (Morgan et al. 1992) and $J$-band 2MASS images of the 2MASS J0559-14 field are shown in Figure 1; no optical counterpart is seen to $B_{J} \sim 23$.

\subsection{Spectral Identification with CorMASS}

Low-resolution, near-infrared spectral data of 2MASS J0559-14 were obtained on 1999 October 24 (UT) using CorMASS. This newly commissioned instrument is an $R \sim 300$ prism cross-dispersed, near-infrared spectrograph, with a $256 \times 256$ NICMOS3 array and a 40 line $\mathrm{mm}^{-1}$ grating, blazed at $4.8 \mu \mathrm{m}$. CorMASS was designed primarily for the spectral classification of candidate low-mass objects color-selected from the 2MASS database. The instrument's echelle format provides simultaneous coverage of the $z J H K$ bands, for $0.8 \lesssim \lambda \lesssim 2.5 \mu \mathrm{m}$. The fixed slit has a width of $2^{\prime \prime}$ and a length of $15^{\prime \prime}$. Further details on this instrument can be found in Wilson et al. (2000).

Conditions during the observations were not photometric, and estimated seeing was $\sim 1 " .5$, with variable thin cirrus throughout the night. Total on-source time was 2400 $\mathrm{s}$ divided into sets of $300 \mathrm{~s}$ integrations, nodding $\sim 5^{\prime \prime}$ along the slit between exposures. Spectra were reduced using standard IRAF routines. After correction for bad pixels, flat field and flux calibration images were corrected for NICMOS3 reset-decay bias (also known as shading) as follows: a quadratic was fit to a row-by-row clipped median of the top quarter of the array, the portion unused by the spectrograph. The fit was extrapolated to 128 rows for the top two quadrants, duplicated for the bottom two quadrants, and subtracted row-by-row from all pixels. All images were flat-fielded with a pixel responsivity solution from the APFLATTEN task using dome flats from all five nights of the run summed together. Nodded image pairs for the science object were subtracted against each other to remove sky background and reset-decay bias. The APALL task was used for spectral extraction. Wavelength calibration was accomplished using spectral observations of the planetary nebula NGC 7027 obtained on 1999 October 25 (UT). Flux calibration was done by dividing by the reduced spectrum of the A2V standard HD 77281 (Elias et al. 1982), which was observed at similar air mass as 2MASS J0559-14 and hand corrected for stellar H Paschen and Brackett recombination lines. The ratio was then multiplied by a $8810 \mathrm{~K}$ (Tokunaga 2000) blackbody to complete the flux calibration. Finally,
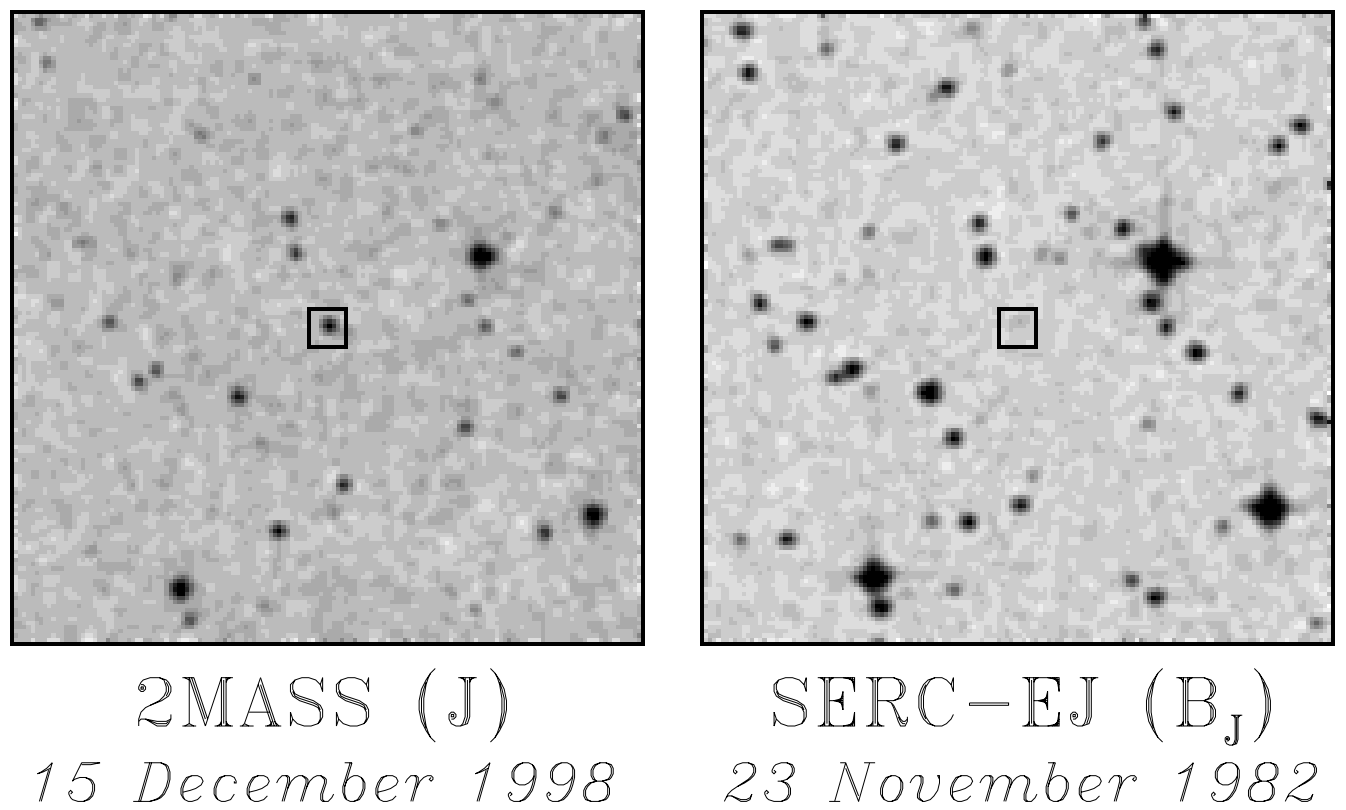

FIG. 1.-SERC-EJ Optical and 2MASS J-band images of the 2MASS J0559-14 field. Images are $5^{\prime} \times 5^{\prime}$ with north up and east to the left. A $20^{\prime \prime} \times 20^{\prime \prime}$ box is drawn around the location of the $\mathrm{T}$ dwarf in both images. 


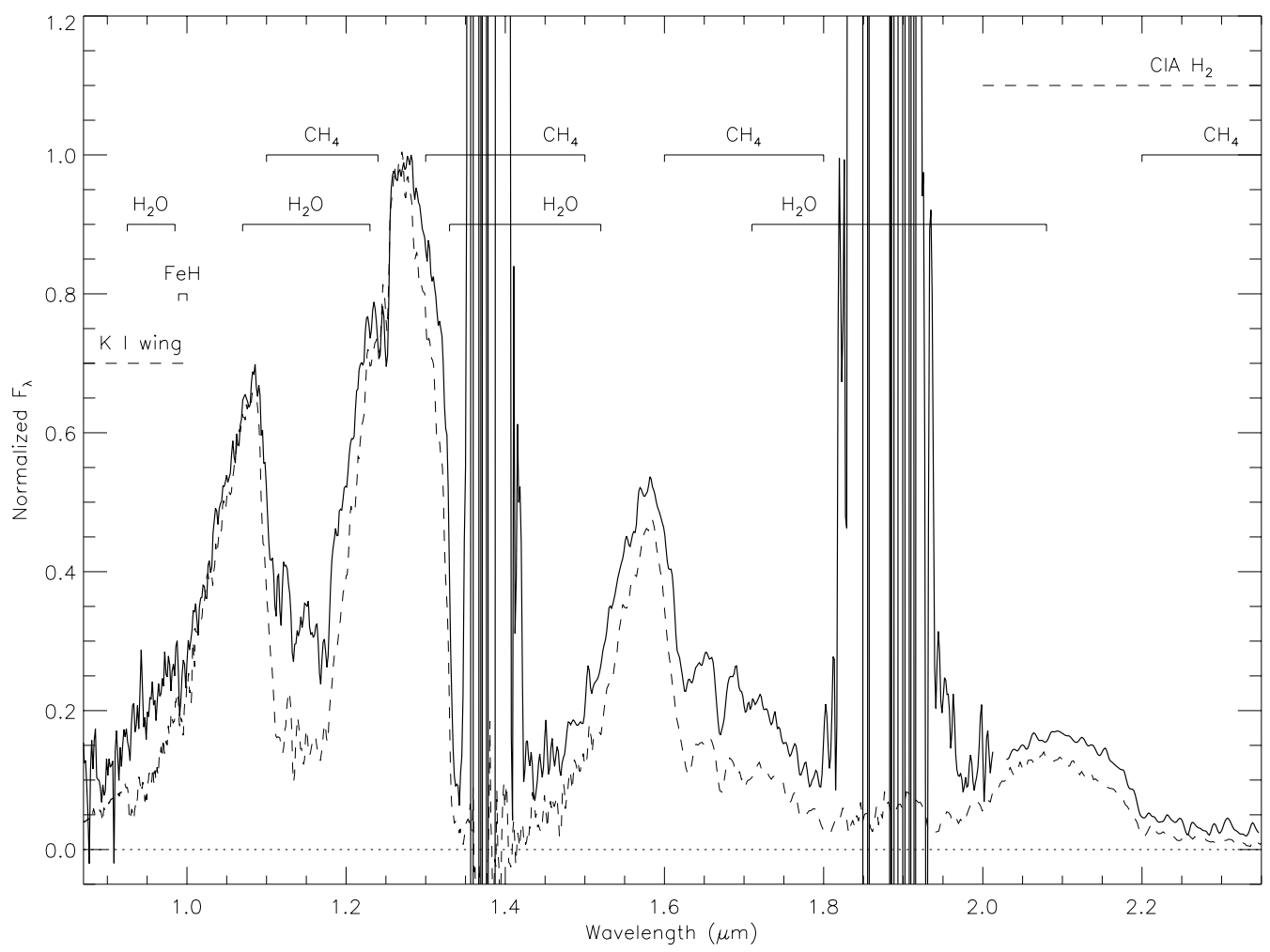

FIG. 2.-Near-infrared spectrum of 2MASS J0559-14 (solid line) obtained by CorMASS. Overlaid are SDSS $1624+00$ data from Burgasser et al. (2000b) and from Strauss et al. (1999) for $0.87-1 \mu \mathrm{m}$ and $1-2.35 \mu \mathrm{m}$, respectively (dashed line). Both spectra are normalized to one at the $1.27 \mu \mathrm{m}$ peak. Prominent molecular bands of $\mathrm{H}_{2} \mathrm{O}, \mathrm{CH}_{4}$, and $\mathrm{H}_{2}$ (collision-induced absorption) are indicated, as is an FeH band at $0.9896 \mu \mathrm{m}$ and the $\mathrm{K} \mathrm{I}$ wing at $z$ band.

all $300 \mathrm{~s}$ observations for each order were weighted by the spectra mean and combined with the SCOMBINE task using a trimmed average. Orders were stitched together with SCOMBINE after hand deletion of noisy data at the ends of the orders.
The spectrum of 2MASS J0559-14 is shown in Figure 2, along with optical $(0.87-1.0 \mu \mathrm{m}$; Burgasser et al. 2000b) and near-infrared (1.0-2.35 $\mu \mathrm{m}$; Strauss et al. 1999) data for SDSSp J162414.37+002915.6 (hereafter SDSS 1624+00). The combined SDSS $1624+00$ spectrum was smoothed to

TABLE 2

NeAR-INFRAREd FeATURes DeteCted in 2MASS J0559-14

\begin{tabular}{|c|c|c|c|}
\hline Feature & $\lambda(\mu \mathrm{m})$ & Transition & Reference for Transition \\
\hline Cs $1 \ldots$ & 0.8943 & $6 s^{2} S_{1 / 2}-6 p{ }^{2} P_{1 / 2}$ & Wiese, Smith, \& Glennon (1966) \\
\hline $\mathrm{K}_{\mathrm{I}} \ldots \ldots . .$. & broadened up to $\sim 0.9$ & $4 s^{2} S_{1 / 2}-4 p^{2} P_{3 / 2,1 / 2}$ & Burrows, Marley, \& Sharp (2000) \\
\hline $\mathrm{H}_{2} \mathrm{O} \ldots \ldots$ & $0.925-0.95$ & $v_{2}=0, v_{1}+v_{3}=3$ & Auman (1967) \\
\hline $\mathrm{H}_{2} \mathrm{O} \ldots \ldots$ & $0.95-0.985$ & $v_{2}=2, v_{1}+v_{3}=2$ & Auman (1967) \\
\hline $\mathrm{FeH} \ldots \ldots$. & 0.9896 & $0-0$ band of $\mathrm{A}^{4} \Delta-\mathrm{X}^{4} \Delta$ & Phillips et al. (1987) \\
\hline $\mathrm{H}_{2} \mathrm{O} \ldots \ldots$ & $1.07-1.11$ & $v_{2}=-1, v_{1}+v_{3}=3$ & Auman (1967) \\
\hline $\mathrm{CH}_{4} \ldots \ldots$ & $1.1-1.24$ & $3 v_{3}$ & Danielson (1966) \\
\hline $\mathrm{H}_{2} \mathrm{O} \ldots \ldots$ & $1.11-1.16$ & $v_{2}=1, v_{1}+v_{3}=2$ & Auman (1967) \\
\hline $\mathrm{H}_{2} \mathrm{O} \ldots \ldots$ & $1.16-1.23$ & $v_{2}=3, v_{1}+v_{3}=1$ & Auman (1967) \\
\hline $\mathrm{K} \mathrm{I} \ldots \ldots$. & 1.1690 & $4 p^{2} P_{0}-3 d^{2} D$ & Wiese, Smith, \& Glennon (1966) \\
\hline $\mathrm{K}_{\mathrm{I}} \ldots \ldots$. & 1.1773 & $4 \mathrm{p}^{2} \mathrm{P}_{0}-3 \mathrm{~d}^{2} \mathrm{D}$ & Wiese, Smith, \& Glennon (1966) \\
\hline $\mathrm{K}$ I $\ldots \ldots$. & 1.2432 & $4 \mathrm{p}^{2} \mathrm{P}_{0}-5 \mathrm{~s}{ }^{2} \mathrm{~S}$ & Wiese, Smith, \& Glennon (1966) \\
\hline $\mathrm{K} \mathrm{I} \ldots \ldots$. & 1.2522 & $4 \mathrm{p}^{2} \mathrm{P}_{0}-5 \mathrm{~s}{ }^{2} \mathrm{~S}$ & Wiese, Smith, \& Glennon (1966) \\
\hline $\mathrm{CH}_{4} \ldots \ldots$ & $1.3-1.5$ & $v_{2}+2 v_{3}$ & Danielson (1966) \\
\hline $\mathrm{H}_{2} \mathrm{O} \ldots \ldots$ & $1.33-1.43$ & $v_{2}=0, v_{1}+v_{3}=2$ & Auman (1967) \\
\hline $\mathrm{H}_{2} \mathrm{O} \ldots \ldots$ & $1.43-1.52$ & $v_{2}=2, v_{1}+v_{3}=1$ & Auman (1967) \\
\hline $\mathrm{CH}_{4} \ldots \ldots$ & $1.6-1.8$ & $2 v_{3}$ & Danielson (1966) \\
\hline $\mathrm{H}_{2} \mathrm{O} \ldots \ldots$ & $1.71-1.80$ & $v_{2}=-1, v_{1}+v_{3}=2$ & Auman (1967) \\
\hline $\mathrm{H}_{2} \mathrm{O} \ldots \ldots$ & $1.80-2.08$ & $v_{2}=1, v_{1}+v_{3}=1$ & Auman (1967) \\
\hline $\mathrm{CH}_{4} \ldots \ldots$ & $2.2-2.6$ & $v_{2}+v_{3}$ & Danielson (1966) \\
\hline $\mathrm{H}_{2} \ldots \ldots \ldots$ & Centered at 2.4 & 1-0 quadrupole (CIA) & Danielson (1966) \\
\hline
\end{tabular}


the resolution of CorMASS. Spectra for both objects are normalized to one at $1.27 \mu \mathrm{m}$ ( $J$-band peak). Close up views of 0.88-1.01 and 1.15-1.345 $\mu \mathrm{m}$ are shown in Figure 3.

\section{DISCUSSION OF THE NEAR-INFRARED SPECTRUM}

\subsection{Spectral Features}

Table 2 summarizes the spectral features detected in 2MASS J0559-14, with identifications from Pearse \& Gaydon (1963), Danielson (1966), Wiese, Smith, \& Glennon (1966), and Phillips et al. (1987). Only major absorption bands of $\mathrm{H}_{2} \mathrm{O}$ and $\mathrm{CH}_{4}$ are tabulated. The characteristic $\mathrm{CH}_{4}$ bands at 1.6 and $2.2 \mu \mathrm{m}$ are present, as are bands at 1.1 and $1.3 \mu \mathrm{m}$ identified from laboratory data (Fink \& Larson 1979) that are blended with $\mathrm{H}_{2} \mathrm{O}$ bands at the same wavelengths. An FeH feature is seen at $0.9896 \mu \mathrm{m}(0-0$ band of $A^{4} \Delta-X^{4} \Delta$ ) which has also been identified in SDSS $1624+00$ (Burgasser et al. 2000b). We do not detect the higher order $0-1 \mathrm{FeH}$ band at $1.191 \mu \mathrm{m}$, which is seen to weaken in the latest L dwarfs (McLean et al. 2000). Two sets of $\mathrm{K}$ I doublets are noted at 1.1690 and $1.1773 \mu \mathrm{m}$ $\left(4 p^{2} P_{0}-3 d^{2} D\right)$ and 1.2432 and $1.2522 \mu \mathrm{m}\left(4 p^{2} P_{0}-5 s^{2} S\right)$, as is Cs I at $0.8943 \mu \mathrm{m}\left(6 s^{2} S_{1 / 2}-6 p^{2} P_{1 / 2}\right)$. We do not detect the 2- $0 X^{1} \Sigma^{+}-X^{1} \Sigma^{+}$band of $\mathrm{CO}$ at $2.3 \mu \mathrm{m}$.

Comparison between 2MASS J0559-14 and SDSS $1624+00$ reveals significant differences in spectral morphology. In Figure 2, it is apparent that the slope between 0.9 and $1.05 \mu \mathrm{m}$ is shallower in 2MASS J0559-14, likely due to decreased absorption by the pressure-broadened $\mathrm{K}$ I doublet at 0.7665 and $0.7699 \mu \mathrm{m}$ (Liebert et al. 2000). $\mathrm{CH}_{4}$ and $\mathrm{H}_{2} \mathrm{O}$ features are generally weaker in 2MASS J0559-14, as noted by the significantly weakened $1.1-1.2$ $\mu$ m trough between $z$ - and $J$-band peaks. The decreased $\mathrm{CH}_{4}$ opacity noticeably affects the shape of the $J$-band peak near $1.27 \mu \mathrm{m}$, as the weak $\mathrm{CH}_{4}$ wings at 1.24 and $1.30 \mu \mathrm{m}$ carve out less flux on either side of the peak. Also in Figure 2 , a significant flux offset is readily apparent at the base of the $1.6 \mu \mathrm{m} \mathrm{CH}$ band in 2MASS J0559-14, and relative enhancement of flux at both $\mathrm{H}$ and $\mathrm{K}$ bands in this object is almost certainly due to decreased $\mathrm{H}_{2}$ CIA (1-0 quadrupole), $\mathrm{H}_{2} \mathrm{O}$, and $\mathrm{CH}_{4}$ opacity.

\subsection{MASS J0559-14 is a Warm T Dwarf}

The weak $\mathrm{CH}_{4}$ bands seen in 2MASS J0559-14 are unique among the current sample of $\mathrm{T}$ dwarfs, and the reduced opacity can be most readily explained if this object is warmer than other known T dwarfs. At higher effective temperatures, the dominant carbon-bearing species changes from $\mathrm{CO}$ to $\mathrm{CH}_{4}$ at small optical depth, so that the $\mathrm{CH}_{4}$ column density will be less than that of cooler $\mathrm{T}$ dwarfs, and observed band strengths correspondingly weaker. Increased thermal flux will also be seen at the base of these bands, particularly at $1.6 \mu \mathrm{m}$, which is unaffected by $\mathrm{H}_{2} \mathrm{O}$ absorption. The lower $\mathrm{CH}_{4}$ column density directly affects the $\mathrm{H}_{2} \mathrm{O}$ column density, via the reaction $\mathrm{CO}+3 \mathrm{H}_{2} \rightarrow \mathrm{CH}_{4}$ $+\mathrm{H}_{2} \mathrm{O}$, leading to shallower bands at 1.1 and $1.45 \mu \mathrm{m}$. Water can also be heated and dissociated by dust layers deep in the photosphere (Leggett, Allard, \& Hauschildt 1998). Finally, decreased $\mathrm{H}_{2}$ CIA opacity at $K$ band, congruous with reduced $\mathrm{CH}_{4}$ and $\mathrm{H}_{2} \mathrm{O}$ opacity, will result in redder $J-K_{s}$ colors with warmer $T_{\text {eff }}$. These features are observed in 2MASS J0559-14, and its warm temperature is independently supported by the detection of the 0.9896 $\mu \mathrm{m} \mathrm{FeH}$ band, which is present but weakening in the latest
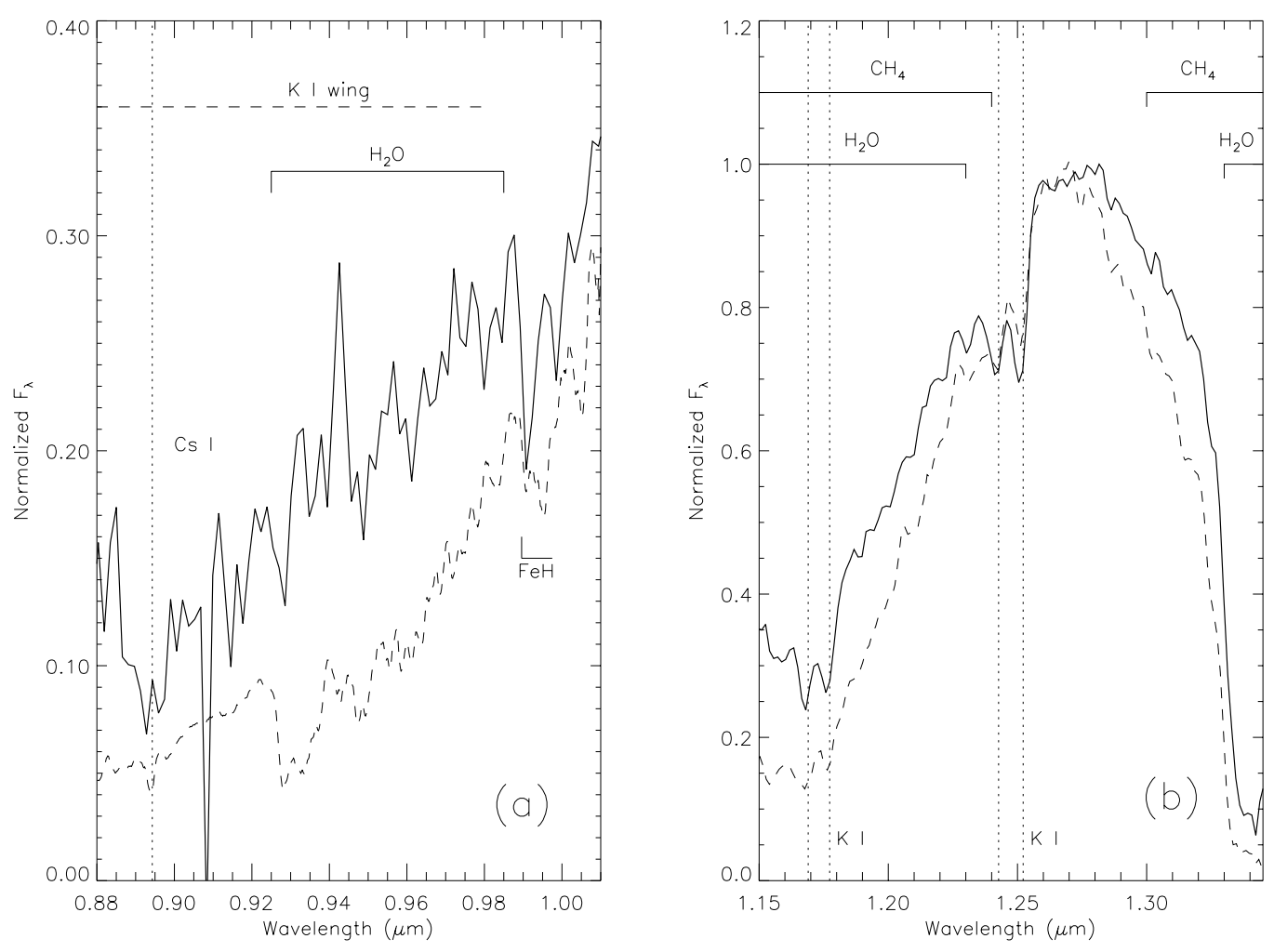

FIG. 3.-Two detailed regions of Figure 2 showing $(a) 0.88-1.01 \mu \mathrm{m}$ and $(b) 1.15-1.345 \mu \mathrm{m}$. Spectra are normalized as in Figure 2. Features of FeH (0.9896 $\mu \mathrm{m}), \mathrm{K} \mathrm{I}(1.1690,1.1773,1.2432,1.2522 \mu \mathrm{m})$, and $\mathrm{Cs} \mathrm{I}(0.8943 \mu \mathrm{m})$ are indicated, as are $\mathrm{H}_{2} \mathrm{O}$ and $\mathrm{CH}_{4}$ bands. 
L dwarfs (Kirkpatrick et al. 1999). A similar argument has been made for SDSS $1624+00$ by Burgasser et al. (2000b), which was one of only three $\mathrm{T}$ dwarfs in that paper to show this feature. SDSS $1624+00$ has been shown to be a warm object via optical continuum measurements between broadened $\mathrm{Na}$ I and $\mathrm{K}$ I features (Liebert et al. 2000), while Nakajima et al. (2000) argue that this object is both warmer and dustier than G1 229B based on its shallower $\mathrm{CH}_{4}$ and $\mathrm{H}_{2} \mathrm{O}$ bands. By analogy, 2MASS J0559-14 should be warmer still. The detection of excited $\mathrm{K}$ I lines at $J$ band, which are seen to weaken in the latest L dwarfs (McLean et al. 2000), is further evidence of the warmth of this object.

The spectral features in 2MASS J0559-14 suggest that it is close to the $L / T$ transition temperature. The lack of $\mathrm{CO}$ detection at $2.3 \mu \mathrm{m}$ is not necessarily contradictory to this hypothesis, as overlying $\mathrm{CH}_{4}$ absorption beyond $2.2 \mu \mathrm{m}$ may mask this weaker feature. Naturally, metallicity, dust, and gravity could also play roles in the band strengths seen in 2MASS J0559-14; however, temperature is likely to be the dominant determinant given the concordance of spectral features as discussed above. We can make a conservative $T_{\text {eff }}$ constraint for this object based on the temperature of Gl 229B, which is clearly cooler, and a temperature estimate of the L8V companion dwarf Gl 584C (Kirkpatrick et al. 2000); this translates into a range of $1000 \mathrm{~K} \lesssim T_{\text {eff }} \lesssim$ $1300 \mathrm{~K}$. Parallax and bolometric luminosity measurements of this object would allow a direct determination of temperature.

\section{THE BRIGHTNESS OF 2MASS J0559-14}

The relative brightness of 2MASS J0559-14 as compared to other $\mathrm{T}$ dwarfs suggests that it may be a nearby brown dwarf. We can estimate its distance using simple scaling arguments based on Gl 229 $\mathrm{B}^{7}$. First, if we assume this object has the same intrinsic luminosity as G1 229B, we estimate its distance to be 4.6 pc. However, 2MASS $\mathrm{J} 0559-14$ is probably a warmer object than G1 $229 \mathrm{~B}$, so it should be more distant. An alternate estimate can be made if we adopt a radius $R \approx R_{\mathrm{G} 1229 \mathrm{~B}}$ and bounding $T_{\text {eff }} \lesssim 1300$ $\mathrm{K}$, so that $L \lesssim 2.2 \times 10^{-5} L_{\odot}$. Using a G1 229B bolometric correction, we obtain $M_{J} \gtrsim 14.2$ and thus $d \lesssim 8.4$ pc. Note, however, that an L8V has $M_{J}=15.0$ (Kirkpatrick et al. 2000), implying that our $\mathrm{BC}_{J}$ value may be too high; indeed, $\mathrm{BC}_{J}$ may be only 1.3 for an L8V (Reid et al. 1999). Adopting a smaller $\mathrm{BC}_{J}$ yields a smaller distance. Therefore, barring multiplicity, 2MASS J0559-14 is probably 5-8 pc distant, and likely falls within the 8 pc nearby star sample defined by Reid \& Gizis (1997).

The brightness of this object allows substantial follow-up over a broad wavelength range, particularly for $\lambda>2.5 \mu \mathrm{m}$, where the fundamental absorption bands of $\mathrm{H}_{2} \mathrm{O}(2.7 \mu \mathrm{m})$, $\mathrm{NH}_{3}(3.0 \mu \mathrm{m}), \mathrm{CH}_{4}(3.3 \mu \mathrm{m}), \mathrm{H}_{2} \mathrm{~S}(3.8 \mu \mathrm{m})$, and $\mathrm{CO}(4.7 \mu \mathrm{m})$ are found. The latter $1-0 \mathrm{X}^{1} \Sigma^{+}-\mathrm{X}^{1} \Sigma^{+}$band of $\mathrm{CO}$ can aid in constraining its atmospheric abundance (Noll, Geballe,

\footnotetext{
${ }^{7}$ In this section we adopt the following values for Gl 229B: $d=5.77 \pm 0.04 \mathrm{pc}$ (Perryman et al. 1997), $T_{\text {eff }}=960 \pm 70 \mathrm{~K}$ (Marley et al. 1996), $\quad R \approx R_{\mathrm{Jup}}=7.1 \times 10^{9} \quad \mathrm{~cm} \quad$ (Burrows \& Liebert 1993), $J=14.32 \pm 0.05, \quad L=(6.6 \pm 0.6) \times 10^{-6} \quad L_{\odot}, \quad$ and $\quad \mathrm{BC}_{J}=2.2 \pm 0.1$ (Leggett et al. 1999).
}

\& Marley 1997), essential in testing hypotheses of mixing (Oppenheimer et al. 1998; Griffith \& Yelle 1999) and transparency (Lodders 1999) in T dwarf atmospheres. Deuterated molecules, such as $\mathrm{CH}_{3} \mathrm{D}$, are of interest for study in the 3-5 $\mu \mathrm{m}$ range, as they yield information on the deuterium burning history of these brown dwarfs. Midinfrared wavelengths $(8-14 \mu \mathrm{m})$ are sensitive to vibration-rotation bands of alkali chlorides and sulfides, which are more abundant than their atomic alkali counterparts at low temperatures and high pressures (Lodders 1999) and may serve as excellent temperature discriminants. This region also contains the strong fundamental $v_{2}$ band of $\mathrm{NH}_{3}(10.5 \mu \mathrm{m})$ and various silicate features.

Additionally, optical spectra shortward of $0.9 \mu \mathrm{m}$ are critical in determining the behavior of the alkalis in cool brown dwarfs, particularly pressure-broadened $\mathrm{Na}$ I $(0.5890$ and $0.5896 \mu \mathrm{m})$ and $\mathrm{K}_{\mathrm{I}}(0.7665$ and $0.7699 \mu \mathrm{m})$ doublets, and Cs I lines at 0.8521 and $0.8943 \mu \mathrm{m}$, which may also be used as temperature discriminants (Reid et al. 2000; Burrows, Marley, \& Sharp 2000; Basri et al. 2000). While these features have been seen in T dwarfs such as SDSS $1624+00$ (Liebert et al. 2000), detailed investigation of Li I $(0.6708 \mu \mathrm{m})$ and $\mathrm{Rb} \mathrm{I}(0.7800$ and $0.7948 \mu \mathrm{m})$ lines have been hampered by the lack of detectable flux at these wavelengths. Investigation in this spectral regime is currently underway.

We thank M. Strauss for the kind use of the SDSS $1624+00$ near-infrared spectrum and acknowledge useful discussions with C. Griffith and M. Marley. We thank our anonymous referee for helpful comments and suggestions. We also recognize the efforts of the 2MASS staff and scientists in creating a truly incredible astronomical resource, and the expert assistance of the Palomar Observatory staff during imaging and spectroscopic observations. A. J. B., J. D. K., and J. E. G. acknowledge the support of the Jet Propulsion Laboratory, California Institute of Technology, which is operated under contract with the National Aeronautics and Space Administration. J. C. W. acknowledges support by NASA grant NAG 5-4376. Observations from Palomar Observatory were made as part of a continuing collaboration between the California Institute of Technology and Cornell University. The Digitized Sky Survey was produced at the Space Telescope Science Institute under grant NAG W-2166. SERC-EJ data were scanned by DSS from photographic data obtained using the UK Schmidt Telescope, operated by the Royal Observatory Edinburgh, with funding from the UK Science and Engineering Research Council. DSS images were obtained from the Canadian Astronomy Data Centre, which is operated by the Herzberg Institute of Astrophysics, National Research Council of Canada. IRAF is distributed by the National Optical Astronomy Observatories, which are operated by the Association of Universities for Research in Astronomy, Inc., under cooperative agreement with the National Science Foundation. This publication makes use of data from the Two Micron All Sky Survey, which is a joint project of the University of Massachusetts and the Infrared Processing and Analysis Center, funded by the National Aeronautics and Space Administration and the National Science Foundation. 
REFERENCES

Arnouts, S., D’Odorico, S., Cristiani, S., Zaggia, S., Fontana, A., \& Giallongo, E. 1999, A\&A, 341, 641

Auman, J., Jr. 1967, ApJS, 14, 171

Basri, G., Mohanty, S., Allard, F., Hauschildt, P. H., Delfosse, X., Martín, E. L., Forveille, T. \& Goldman, B. 2000, ApJ, in press

Burgasser, A. J., et al. 1999, ApJ, 522, L65

.2000a, ApJ, 531, L57

Burgasser, A. J., Kirkpatrick, J. D., Reid, I. N., Liebert, J., Gizis, J. E., \& Brown, M. E. 2000b, AJ, 120, in press

Burrows, A., \& Liebert, J. 1993, Rev. Mod. Phys., 65, 301

Burrows, A., Marley, M. S., \& Sharp, C. M. 2000, ApJ, 531, 438

Burrows, A., \& Sharp, C. M. 1999, ApJ, 512, 843

Cuby, J. G., Saracco, P., Moorwood, A. F. M., D’Odorico, S., Lidman, C., Comerón, F., \& Spyromilio, J. 1999, A\&A, 349, L41

Danielson, R. E. 1966, ApJ, 143, 949

Elias, J. H., Frogel, J. A., Matthews, K., \& Neugebauer, G. 1982, AJ, 87, 1029

Fegley, B., Jr., \& Lodders, K. 1996, ApJ, 472, L37

Fink, U., \& Larson, H. P. 1979, ApJ, 233, 1021

Griffith, C. A., \& Yelle, R. V. 1999, ApJ, 519, L85

Gunn, J. E., \& Weinberg, D. H. 1995, in Wide-Field Spectroscopy and the Distant Universe, ed. S. Maddox \& A. Aragón-Salamanca (Singapore: World Scientific), 3

Kirkpatrick, J. D., et al. 1999, ApJ, 519, 802

Kirkpatrick, J. D., et al. 2000, AJ, 120, in press

Leggett, S. K., Allard, F., \& Hauschildt, P. H. 1998, ApJ, 509, 836

Leggett, S. K., Toomey, D. W., Geballe, T. R., \& Brown, R. H. 1999, ApJ, 517, L139

Liebert, J., Reid, I. N., Burrows, A., Burgasser, A. J., Kirkpatrick, J. D., \& Gizis, J. E. 2000, ApJ, 533, L155

Lodders, K. 1999, ApJ, 519, 793

Marley, M. S., Saumon, D., Guillot, T., Freedman, R. S., Hubbard, W. B., Burrows, A., \& Lunine, J. I. 1996, Science, 272, 1919
McLean, I., et al. 2000, ApJ, 533, L45

Morgan, D. H., Tritton, S. B., Savage, A., Hartley, M., \& Cannon, R. D. 1992, in Digitised Optical Sky Surveys, ed. H. T. MacGillivray \& E. B. Thomson (Dordrecht: Boston), 11

Murphy, D. C., Persson, S. E., Pahre, M. A., Sivaramakrishnan, A., \& Djorgovski, S. G. 1995, PASP, 107, 1234

Nakajima, T., Oppenheimer, B. R., Kulkarni, S. R., Golimowski, D. A., Matthews, K., \& Durrance, S. T. 1995, Nature, 378, 463

Nakajima, T., Tsuji, T., Maihara, T., Iwamuro, F., Motohara, K., Taguchi, T., Hata, R., Tamura, M., \& Yamashita, T. 2000, PASJ, 52, 87

Noll, K. S., Geballe, T. R., \& Marley, M. S. 1997, ApJ, 489, L87

Oppenheimer, B. R. 1999, Ph.D. thesis (Pasadena: Caltech)

Oppenheimer, B. R., Kulkarni, S. R., Matthews, K., van Kerkwijk, M. H. 1998, ApJ, 502, 932

Pearse, R. W. B., \& Gaydon, A. G. 1963, The Identification of Molecular Spectra (3d ed.; New York: Wiley)

Perryman, M. A. C., et al. 1997, A\&A, 323, L49

Phillips, J. G., Davis, S. P., Lindgren, B., \& Balfour, W. J. 1987, ApJS, 65, 721

Reid, I. N., et al. 1999, ApJ, 521, 613

Reid, I. N., \& Gizis, J. E. 1997, AJ, 113, 2246

Reid, I. N., Kirkpatrick, J. D., Gizis, J. E., Dahn, C. C., Monet, D. G., Williams, R. J., Liebert, J., \& Burgasser, A. J. 2000, AJ, 119, 369

Skrutskie, M. F., et al. 1997, in The Impact of Large-Scale Near-IR Sky Surveys, ed. F. Garzon (Dordrecht: Kluwer), 25

Strauss, M. A., et al. 1999, ApJ, 522, L61

Tokunaga, A. T. 2000, in Allen's Astrophysical Quantities, ed. A. N. Cox (4th ed.; New York: Springer), 151

Tsvetanov, Z. I., et al. 2000, ApJ, 531, L61

Wiese, W. L., Smith, M. W., \& Glennon, B. M. 1966, Atomic Transition Probabilities, Vol. 1. (Washington, D. C.: GPO)

Wilson, J. C., et al. 2000, in preparation 